\title{
Cultura mundial y mundos de significado culturalmente específicos
}

\section{Cultura mundial e mundos de significado culturalmente específicos}

\section{World Culture and culturally specific worlds of meaning}

Jürgen Schriewer ${ }^{1}$

\begin{abstract}
RESUMEN
"Cultura mundial" no es - sobre todo en el título de un ensayo - un concepto de alcance modesto, ni carece tampoco de contenidos teóricos elaborados. Requiere, por tanto, de unas aclaraciones previas. En consecuencia, abriré este trabajo con un delineamiento del concepto de "cultura mundial", así como del horizonte teórico del que está tomado. Se trata de un marco teórico que intenta comprender y explicar los procesos del creciente entrelazamiento de las relaciones de comunicación e intercambio que se está llevando a cabo a nivel mundial y en casi todos los ámbitos sociales, económicos y científico-tecnológicos. En la segunda parte, voy a presentar algunas líneas de investigación de la sociología y la educación comparadas, cuyos resultados apuntan en una dirección opuesta a las tesis desarrolladas por los teóricos de la "cultural mundial". Por consiguiente, en la tercera parte, pasaré al concepto alternativo de "mundos de significado culturalmente específicos", para, en la cuarta y última parte, abordar brevemente el actual debate entre teorías "de la convergencia" y "de la divergencia", y plantear algunas expectativas respecto de una conceptualización que, a partir de su capacidad explicativa, tenga el potencial de superar tales concepciones opuestas.
\end{abstract}

Palabras-clave: Cultura mundial; mundos de significado culturalmente específicos; sociedad moderna.

${ }^{1}$ Humboldt-Universität zu Berlin. Geschwister-Scholl-Straße 7. 10117 Berlin, Deutschland. 


\title{
RESUMO
}

Cultura mundial não é - sobretudo em um título de um ensaio - um conceito de alcance modesto e tampouco necessita de elaborados conteúdos teóricos. Portanto, requer alguns esclarecimentos prévios. Nesse sentido, iniciarei este artigo com um delineamento do conceito de "cultura mundial", bem como do horizonte teórico de que ele tem feito parte. Trata-se de um marco teórico que procura compreender e explicar os processos do crescente entrelaçamento das relações de comunicação e intercâmbio que estão acontecendo em nível mundial em quase todos os âmbitos sociais, econômicos e científico-tecnológicos. Na segunda parte, apresentarei algumas linhas de investigação da sociologia e da educação comparadas, cujos resultados apontam em uma direção oposta às teses desenvolvidas pelos teóricos da "cultura mundial". Na sequência, utilizarei na terceira parte o conceito alternativo de "mundos de significado culturalmente específicos", e na quarta e última parte, abordarei brevemente o atual debate entre as teorias "da convergência" e "da divergência" ao levantar algumas expectativas de uma conceituação que a partir de seu poder explicativo tem potencial para superar esses pontos de vista opostos.

Palavras-chave: cultura mundial; mundos de significado culturalmente específicos; sociedade moderna.

\begin{abstract}
World Culture is not - especially in the title of an essay - a concept of modest scope, nor does it require elaborate theoretical content. Therefore, it requires some previous explanation. In this direction, I will begin this article with an outline of the concept of "world culture", as well as of the theoretical horizon it has been a part of. It is a theoretical framework that seeks to understand and explain the processes of growing, interweaving relations of communication and exchange that are happening worldwide in almost all spheres - social, economic, scientific and technological. In the second part, I will present and compare a few lines of investigation in Sociology and Education, the results pointing in an opposite direction than the thesis developed by theorists of "world culture". Following that, I will utilize the third part for the alternative concept of "culturally specific worlds of meaning", and in the fourth and final part, I will briefly discuss the current debate between the "convergence" and "divergence" theories in raising some expectations of a conceptualization that, from its explanatory power, has the potential to overcome these opposite points of view.
\end{abstract}

Keywords: World Culture; culturally specific worlds of meaning; modern society. 
"Cultura mundial" no es - sobre todo en el título de un ensayo - un concepto de alcance modesto, ni carece tampoco de contenidos teóricos elaborados. Requiere, por tanto, de unas aclaraciones previas. En consecuencia, abriré este trabajo con un delineamiento del concepto de "cultura mundial", así como del horizonte teórico del que está tomado. Se trata de un marco teórico que intenta comprender y explicar los procesos del creciente entrelazamiento de las relaciones de comunicación e intercambio que se está llevando a cabo a nivel mundial y en casi todos los ámbitos sociales, económicos y científico-tecnológicos. En la segunda parte, voy a presentar algunas líneas de investigación de la sociología y la educación comparadas, cuyos resultados apuntan en una dirección opuesta a las tesis desarrolladas por los teóricos de la "cultural mundial". Por consiguiente, en la tercera parte, pasaré al concepto alternativo de "mundos de significado culturalmente específicos", para, en la cuarta y última parte, abordar brevemente el actual debate entre teorías "de la convergencia" y "de la divergencia", y plantear algunas expectativas respecto de una conceptualización que, a partir de su capacidad explicativa, tenga el potencial de superar tales concepciones opuestas.

\section{El concepto de "cultura mundial" y el neoinstitucionalismo sociológico}

"Cultura mundial" es la denominación general y abarcadora que se da a una línea de investigación y teorización sociológica que va cobrando cada vez más prominencia. Se trata del neoinstitucionalismo sociológico, tanto en su versión teórica inicial, formulada por John W. Meyer y Brian Rowan, como en las variantes que el mismo Meyer ha venido desplegando en colaboración con Francisco O. Ramirez y un grupo creciente de doctorandos y jóvenes investigadores. El concepto sirve como una especie de abreviatura que engloba un programa de investigación, en el marco del cual se ha desarrollado una vasta amplitud de miras, cuya intención no sólo es la de captar de modo teórico la dinámica de la conformación de una sociedad mundial y de las estructuras que le son características, sino también de dar fundamento empírico a las tesis elaboradas dentro de este programa, valiéndose para ello de un gran número de investigaciones de índole cuantitativo-comparada ${ }^{2}$.

Como consecuencia de sus premisas, que proceden de la sociología de las organizaciones y del conocimiento, el concepto neoinstitucionalista de "cultura

${ }^{2}$ Cf. (KRÜCKEN; DRORI, 2009). Véase también la reciente traducción de una selección de los ensayos más importantes sobre la educación publicada como (MEYER; RAMIREZ, 2011). 
mundial" entiende la sociedad moderna como un orden primordialmente cultural, es decir, constituido tanto a partir de pautas cognitivas y asunciones compartidas colectivamente como de los modelos de orientación y expectativas que resultan de las primeras. La sociedad moderna se constituye, entonces, como un conjunto de interpretaciones de la realidad (accounts en la terminología inglesa), de ideas directrices de regulación (rules) y de programas de acción estrechamente conexos a ellas (ideologies). Estas tres componentes - interpretaciones de lo social, ideas directrices y programas de acción - son construcciones socio-comunicativas que ya no dan lugar a dudas, sino que se aceptan como naturales y legítimas, vale decir, en este sentido, como "institucionalizadas" o "endosadas". Están, por un lado, insertas en la tradición occidental de los procesos de racionalización y secularización que cobraron una dinámica particular a partir del Renacimiento y la Reforma. Por otro lado, se han ido desarrollando en un sentido universalista y han ganado una asombrosa validez y aceptación mundial en el transcurso de las últimas décadas del siglo XX. Entre estos "mitos racionalizados" - como también los llaman Meyer y sus colaboradores, en un arranque de ironía - figuran, en primerísimo lugar, las ideas rectoras del desarrollo individual de la personalidad y de la condición de ciudadano, del progreso social y el desarrollo económico, así como también del estado como marco legítimo y garante de una sociedad nacional y sus actividades ${ }^{3}$. Desde el punto de vista del neoinstitucionalismo, la "globalización" no significa más que los procesos de difusión por los que se diseminan al nivel mundial tales interpretaciones de la realidad e ideas rectoras, dotadas, además, de la capacidad de orientar a las decisiones de los actores como lo harían "guiones" (scripts). Estos procesos de difusión se ven constantemente dinamizados por un sinnúmero de interacciones, referencias e imitaciones entre sociedades, así como por la construcción de modelos de reforma o mejoramiento abstractos o - según los términos del mismo John Meyer - "teorizados" (STRANG; MEYER, 1993). Fue, ante todo, a partir de la segunda mitad del siglo XX que estas formas y vías de difusión comenzaron a intensificarse en un entramado comunicativo en el que las redes regionales e interregionales de científicos y expertos, así como también las organizaciones internacionales gubernamentales y no gubernamentales, interactúan - mediante congresos, publicaciones, declaraciones y programas de desarrollo - con una intensidad nunca antes vista, manteniendo e incluso potenciando la marcha de un proceso autosustentado de "construcción social de la realidad" a nivel mundial ${ }^{4}$. La "cultura mundial" pasa entonces a entenderse como el conjunto de estruc-

3 Véase, en tal sentido, (RAMIREZ; BOLI-BENNETT, 1987).

${ }^{4}$ Formulación basada en (BERGER; LUCKMANN, 1976). Cf., en especial, (BOLI; THOMAS, 1999), así como también (FUCHS; SCHRIEWER, 2007). 
turas de expectativas mundialmente reconocidas y de sistemas de significado racionalizado, que, en virtud de su legitimidad cada vez más universalizada, pueden arrogarse la pretensión de servir para la estructuración de las conductas tanto de los individuos como de las organizaciones o los estados. A diferencia de la "economía mundial" que, según Wallerstein, surge de la dinámica de incorporación expansionista propia del sistema capitalista, la "cultura mundial" concebida en términos neoinstitucionalistas constituye un "entorno cultural trasnacional" que ha llevado, en numerosos campos de la actividad social - de la organización política y los regímenes de ciudadanía, pasando por principios jurídicos y derechos humanos, hasta llegar a la protección del medio ambiente, la racionalidad científica y la educación -, a una conformación de estructuras "isomorfas" a nivel mundial ${ }^{5}$.

Como ya resulta de lo expuesto, las tesis sustentadas por el enfoque neoinstitucionalista conceden especial atención a la "institucionalización" de un programa mundial centrado en las interrelaciones entre la educación y el desarrollo ${ }^{6}$. Es este programa el que, a la manera de un guión (script), informa las ideas de los actores competentes - políticos, planificadores y administradores, así como también asociaciones, autoridades estatales y parlamentos $-\mathrm{y}$ da dirección a su actividad política. Este programa mundial de educación y desarrollo se fue traduciendo con intensidad creciente en el curso del siglo XX, tanto en los principios jurídicos generales como en los objetivos de las políticas educativas y de desarrollo consignados en las constituciones y las legislaciones de países de todos los continentes por igual. Ha logrado obtener - siempre según Meyer y sus colaboradores - el rango de una visión cultural del mundo moderno que despoja de su legitimidad y marginaliza otras interpretaciones y modelos de organización alternativos. Este programa mundial, como visión cultural, está dotado de una pretensión de explicación y una validez de carácter vinculante, en virtud de la que es capaz de acompañar, fomentar y potenciar los procesos tendientes al desarrollo y a la reforma educativa que tienen lugar en todos los continentes. Las tesis formuladas por el neoinstitucionalismo se ven corroboradas por un número ya casi inabarcable de análisis cuantitativos concebidos en la mayoría de los casos con el objetivo de indagar los fenómenos estudiados por medio de comparaciones tanto inter temporales como internacionales. Sobre este fundamento los autores neoinstitucionalistas logran brindar

5 Cf. los volúmenes muy representativos de (THOMAS; MEYER; RAMIREZ; BOLI, 1987), y (DRORI; MEYER; RAMIREZ; SCHOFER, 2003). Para una discusión comentada respecto del neoinstitucionalismo, véanse también (HASSE; KRÜCKEN, 2005).

6 Una "world level developmental cultural account and educational ideology" como la especifican (FIALA; LANFORD, 1987). 
explicaciones, sólidamente basadas en lo empírico y estimulantes en lo teórico, tanto de la diseminación mundial y la estructuración "isomorfa" de los modelos "racionalizados" de la organización educativa y los currículos escolares, como de los procesos convergentes de expansión masiva que se verifican en todos los niveles de los sistemas educativos completamente desarrollados ${ }^{7}$.

\section{"Tendencias contrarias": los resultados de la educación y la sociología comparadas}

Sin embargo, dejando de lado todo lo estimulantes que puedan ser sus contenidos y la plausibilidad de sus explicaciones, las tesis neoinstitucionalistas también han sido, por cierto, contradichas. Claro está que contradicciones análogas entre la modelización teórica y los hallazgos empíricos se aplican también a otras teorías alternativas, por ejemplo a aquellas que, partiendo de análisis críticos de la historia económica, afirman la emergencia de un único sistema mundial de carácter capitalista ${ }^{8}$. Como he resumido en otro lugar,Schriewer (2011), a la luz de distintas perspectivas conceptuales y tomando como referencia distintas temáticas, la educación y la sociología comparadas han logrado poner en claro que los procesos sociales de entrelazamiento mundial o de - en los términos del neoinstitucionalismo - "isomorfización" según las pautas de una cultura mundial no se realizan en modo alguno de manera lineal, teleológica o evolucionista. Tales procesos constituyen, antes bien, situaciones complejas y variables de "globalización" y "fragmentación" (MENZEL, 1998; CLARK, 1997) o de "integración" y "impugnación” (contestation). (STROMQUIST; MONKMAN, 2000). Están marcados por el cambio social global (global social change) y la dependencia reiterada de su realización de las distintas trayectorias de desarrollo que se han dado en los diversos contextos nacionales (national path dependencies) (MAYER, 2001). Lo que, al parecer, está caracterizando la realidad social, que supuestamente corresponde con un "sistema mundial", son precisamente las tendencias contrarias de, por un lado, un universalismo al nivel de la concepción abstracta de modelos, y, por el otro, una estructuración generatriz de desviaciones al nivel de los procesos sociales concretos. En tal

7 Véanse, por ejemplo, los trabajos de (BOLI, 1989); (BOLI; RAMIREZ, 1986); (MEYER; KAMENS; BENAVOT, 1992); así como la brillante síntesis de sus diversos trabajos que presentan (MEYER; RAMIREZ, 2002).

${ }^{8}$ Cf., en especial, (WALLERSTEIN, 2004); (WALLERSTEIN, 1974-1989). 
sentido, la posición sustentada por la investigación social comparada internacional podía resumirse, ya a finales del siglo XX, como una "interpenetración" entre las tendencias hacia la globalización económica y las tendencias contrarias hacia la fragmentación sociocultural (FRIEDMAN, 1994).

Corresponde, pues, a tales posiciones que los estudios realizados en distintos campos de la política laboral, educativa y de desarrollo - incluso si se limitan a los países industrializados europeos u occidentales altamente desarrollados - reúnen sus resultados en tramas entrelazadas de "convergencia y divergencia", que varían según el caso estudiado9. Ninguno de los recientes desarrollos que parecen, en cierto sentido, estar promoviendo la "isomorfía" - trátese de la intensificación a nivel internacional de programas de reforma educativa, el enorme incremento de las observaciones, referencias y préstamos de políticas (policies) entre estados, la creciente influencia de las organizaciones transnacionales o internacionales (pensemos, por ejemplo, en el Banco Mundial o en la Organización para la Cooperación y el Desarrollo Económico) o incluso de los desafíos análogos que plantean las fuerzas globales - ninguno de estos desarrollos parece haber dado lugar, según documentan los estudios mencionados, a una convergencia de carácter sostenido ni en el plano de las políticas educativas efectivamente puestas en práctica en diversos países ni tampoco en el plano de las estructuras y prácticas de la educación y la formación profesional. Por eso, los autores de una encuesta bastante exhaustiva llevada a cabo al nivel europeo concluyen:

Tal conclusión no debe del todo sorprendernos. La educación y la formación profesional se encuentran profundamente arraigadas en sus contextos políticos y nacionales. [...] Están estrechamente integradas, según el país, en otros sistemas sociales. Sus formas de administración y control son reflejo de la cultura política dominante, sus currículos han emergido de los saberes específicos y las tradiciones culturales de cada país; sus pedagogías dominantes ponen de manifiesto patrones que se han establecido a lo largo de siglos de experimentación educativa. [...] Así, los sistemas educativos son tan variados como lo es la historia de los países europeos. Llevan inscritos en sí las distintas vías de modernización que cada país ha asumido y operan todavía como talismanes de las particularidades nacionales. Como se ha mostrado en el presente trabajo, cada sistema educativo ha preservado su particularidad, pese a las fuerzas del contexto y de las políticas educativas que obran en conjunto sobre ellos (GREEN; WOLF; LENEY, 1999).

9 (LANGLOIS, 1994); (GREEN; WOLF; LENEY, 1999); (JÜRGENS, 2001). 
Incluso el denominado proceso de Boloña, tan importante para la reestructuración de los programas de estudio y los títulos de la enseñanza superior en Europa, provee un ejemplo instructivo que demuestra la superposición contrarrestante y la trasmutación interpretativa de un universalismo abstracto al nivel de un modelo diseminado a lo largo del continente y los procesos de estructuración generadores de desviaciones a nivel de las implementaciones institucionales concretas (PALOMBA, 2008; SCHRIEWER, 2009)

A más de esto, cuanto mayor es el énfasis que los estudios aquí citados ponen en abordar las distintas teorías del creciente entrelazamiento al nivel mundial - sea la teoría neoinstitucionalista de la "cultura mundial" o la teoría del "sistema mundial" basada en el análisis crítico de la historia económica o, por fin, los modelos de la "sociedad mundial" desarrollados en el marco de la teoría de la diferenciación, (LUHMANN, 2006, pp. 108ss. y 639ss) - tanto más se ven en la obligación ya sea de destacar las múltiples tensiones que genera la presencia simultánea de "ciertos modelos escolares que se difunden al nivel global" y "los significados específicos que estos mismos modelos toman en diferentes contextos socioculturales" 10 , o de hacer resaltar los contrastes que existen entre la isomorfía formal de los modelos de organización estatal, que gozan de aceptación casi normativa a nivel mundial, y las variedades de poder político "informalizadas", "privatizadas" o "intermedias", tal como se practican efectivamente, sobre todo en países del Hemisferio Sur ${ }^{11}$. Otras investigaciones similares, al averiguar las reacciones a la expansión imperialista de Europa o la difusión transcontinental de modelos occidentales de la organización política, han aguzado nuestra vista respecto de la relación, eminentemente dialéctica, que existe entre una occidentalización "de superficie" al nivel de los cambios institucionales y la persistencia de pautas culturalmente específicas al nivel de la interpretación y la elaboración de significados (ROTHERMUND, 1999). Estas investigaciones han puesto de relieve, pues, el complejo entramado de "universalización malograda y desviación creadora" que dicha relación dialéctica arroja como resultado general. (BADIE, 1992b) Son resultados que, evidentemente, confirman conclusiones formuladas ya en otro orden de ideas: Existe un universalismo abstracto de modelos difundidos transnacionalmente que se descompone en pautas estructurales multiformes allí donde tales modelos interactúan, en el transcurso de la puesta en práctica institucional, con diferentes estructuras definidas estatalmente, con regulaciones legales y administrativas, con formas de la división del trabajo en la sociedad, con culturas académicas

${ }^{10}$ (ANDERSON-LEVITT, 2003) y, como complemento, la discusión que de ello hace (STEINER-KHAMSI, 2005).

${ }^{11}$ Cf., por ejemplo, (SCHLICHTE, 2005). 
nacionales, con significados sociales vinculados al contexto, y con visiones religiosas del mundo (SCHRIEWER, 2011, p. 79).

De modo análogo, los análisis que intentan dilucidar la circulación transcontinental de conocimientos y modelos de organización en espacios históricos y regionales particularmente amplios han logrado dar lugar a un gran número de resultados que ilustran la estrecha interrelación que existe entre una "dinámica global de la difusión" (de tales conocimientos y modelos) y su "apropiación contextualmente específica" (SCHRIEWER; CARUSO, 2005). No sólo pueden aplicarse tales hallazgos a la difusión y recepción del llamado "método Bell-Lancaster", que constituyó, en la primera mitad del siglo XIX, el primer ejemplo de la institucionalización a escala mundial de un sistema de enseñanza elaborado y teorizado (CARUSO, 2010), sino corresponden también, por ejemplo, a la recepción, la interpretación y la apropiación, en diferentes contextos político-culturales, del pensamiento filosófico y pedagógico de un clásico del siglo XX tal como John Dewey ${ }^{12}$. De modo característico, la difusión transcultural del conocimiento, los modelos de organización, las pautas o políticas de resolución de problemas, encuentran una reinterpretación y unos procedimientos de adaptación específicos por parte de los grupos culturales o nacionales que los reciben. Como consecuencia de ello, los modelos ofrecidos transculturalmente son seleccionados, en este nuevo ambiente, según los intereses prevalecientes, adaptados a situaciones y necesidades específicas, reinterpretados de acuerdo con líneas culturales y, en grados históricamente diversos, transmutados en reformas estructurales. La multitud de "lógicas de adaptación" insinuadas por numerosos ejemplos y las subyacentes estructuras culturales generadoras de desviación que subyacen a las mismas, las diferentes nociones de tiempo y verdad y las experiencias colectivas sedimentadas en el transcurso de la historia

[...] reducen a puro estado ilusorio las visiones altamente ideológicas que proclaman el fin de la historia [debido a la convergencia supuestamente irresistible hacia el modelo occidental de sociedad capitalista liberal]. Tales puntos de vista pueden aplicarse, en rigor, a fenómenos que ocurren a nivel superficial, así como a la impresión de occidentalización que se desprende de ciertos procesos de importación. Pero, en realidad, por detrás de ese escaparate se encuentra disimulado un complejo juego de importaciones y apropiaciones, y también el resurgimiento de modos populares de acción política y de culturas antiguas, que el comparatista está obligado a tener en cuenta: las trayectorias china, india o japonesa

12 (BRUNO-JOFRÉ; SCHRIEWER, 2012). Una versión castellana menos elaborada ha sido publicada como (BRUNO-JOFRÉ; SCHRIEWER, 2009). 
aparecen configuradas tanto por superposiciones puras, como por apropiaciones moderadas y por la reactualización de tradiciones culturales milenarias (BADIE, 1992a).

A la luz de los corpus de investigación y los resultados correspondientes previamente resumidos, no va a sorprender que hasta las tesis sustentando un supuesto "programa mundial centrado en las interrelaciones entre la educación y el desarrollo"13 hayan sido puestas en duda. En este punto, me permito hacer referencia a un proyecto de investigación comparado llevado a cabo hace pocos años sobre el tema de "Construcción de internacionalidades" (SCHRIEWER, 2007; SCHRIEWER; MARTINEZ, 2007). Su objetivo era explorar las dimensiones y el alcance de la internacionalización del conocimiento pedagógico (tanto en el marco académico como en él de la política educativa). Al nivel de su conceptualización teórica, este proyecto se guió por el modelo de la "autorreflexión sistémica" de los diferentes subsistemas de la sociedad por un lado y del concepto afín de "externalización" por otro. Ambos conceptos, desarrollados por Niklas Luhmann en el conjunto de su teoría general de sistemas sociales autorreferenciales, invitan a dilucidar, no tanto los procesos de difusión y diseminación destacados por el enfoque neoinstitucionalista, sino más bien los mecanismos y coyunturas de adopción que, supuestamente, funcionan en los diversos contextos de reflexión nacional incluidos en la investigación. Como se ilustra en la metáfora de la compuerta, utilizada para explicar más estos mecanismos, las externalizaciones sólo hacen accesible el ambiente internacional de un sistema y sus antecedentes históricos de una forma filtrada, de acuerdo - en otras palabras - con las cambiantes necesidades internas de "significado suplementario"14. Puesto que estas últimas no sólo difieren de una nación a otra sino incluso, dentro de una misma sociedad nacional, de un período histórico y social a otro, implican por tanto no sólo construcciones, sino también desconstrucciones y reconstrucciones de lo internacional. Como consecuencia de ello, el concepto de externalización exige prestar una atención particular, dentro de los discursos educativos de las diferentes naciones o sociedades, a la recepción selectiva, la transformación interpretativa y las distintas modalidades de apropiación de los cuerpos de conocimiento, los modelos pedagógicos y las políticas educativas que se han figurado a nivel internacional. Tales suposiciones teóricas también tienen un impacto decisivo para determinar las unidades analíticas relevantes

${ }^{13}$ El "world level developmental cultural account and educational ideology" como lo especifican (FIALA; LANFORD, 1987).

${ }^{14}$ Cf. las explicaciones más detalladas por (SCHRIEWER, 1993). 
para la investigación comparada. Debido a su postura difusionista, los autores neoinstitucionalistas generalmente han favorecido los diseños de investigación, teniendo en cuenta, si había datos, todas las entidades políticas reconocidas como Estados-nación (por ejemplo, a los Estados miembros de la Organización de las Naciones Unidas o de la Organización de las Naciones Unidas para la Educación). Los análisis del proyecto aquí referido, por el contrario, han adoptado un enfoque alternativo. El examen crítico que se pretende en estos análisis se basa en una selección de unidades de comparación que, aun siendo menores en número, representan una gama máxima de variación sociocultural, política y económica. Por consiguiente, los análisis en cuestión se han concentrado en unidades analíticas que muestran un perfil de civilización característico, que se ha construido a lo largo de prolongados períodos de tiempo y que es subrayado, inter alia, por el poder político y/o el peso demográfico. Como casos ejemplares en este sentido fueron seleccionados España (representando a la Europa occidental latina y católica), Rusia/ la Unión Soviética (representando a la Europa oriental ortodoxa), y China (representando al Asia oriental de tradición confuciana).

Los resultados de estos análisis ponen de manifiesto la existencia de considerables fluctuaciones, integradas dentro de los desarrollos discursivos especíicos de cada país, entre períodos de apertura internacional y aquellos otros de retraimiento sociocéntrico. Revelan, además, entre esos espacios discursivos, diferencias no menos notables relativas a las sociedades de referencia o "visiones del mundo" ideadas en cada caso. Ahora bien, estas fluctuaciones y diferencias son respectivamente más amplias y más profundas de lo que resultaría compatible con las tesis del modelo neoinstitucionalista. Ni siquiera la inclusión explícita de series de datos históricos en el diseño de la investigación consiguió revelar líneas de convergencia claras. Antes bien, lo que los resultados de las investigaciones aquí expuestas ponen de intenso relieve es el hecho de que las tendencias de desarrollo, los modelos de organización educativa, las opciones de reforma y los cuerpos de conocimiento pedagógico-filosófico que han adquirido forma a nivel internacional, así como también la adopción abstracta de "sociedades de referencia" y "world class countries", son refractados por estructuras de selección y por las necesidades de interpretación que son, a su vez, el resultado de fuerzas y tradiciones que vienen determinadas por factores culturales, religiosos, políticos e ideológicos intrínsecos a cada una de las sociedades estudiadas. En contraste con las potencialidades de un enfoque meramente difusionista, es el concepto de externalización el que posee la capacidad no sólo para descifrar las construcciones semánticas generadas en la reflexión educativa (y por medio de la misma), sino también para explicar los vínculos, mediados por las expectativas cambiantes de legitimación y orientación - esto es, por las necesidades, también cambiantes, de un "significado suplementario"-, que unen tales construcciones 
semánticas con los escenarios histórico-político-culturales característicos de las sociedades estudiadas durante sus sucesivos períodos de evolución interna. En otras palabras, el patrón básico que subyace a los hallazgos brevemente resumidos está gobernado más bien por la socio-lógica de la externalización, antes que por la dinámica evolutiva que conduce a la "estandarización" de una ideología educativa a nivel mundial ${ }^{15}$.

En consecuencia, tal y como deja claro un corpus impresionante de investigaciones comparadas e internacionales, los fenómenos contrarios de internacionalización y elaboración estructural específica de cada nación no se producen simplemente de modo colateral, sin relación entre sí. Se hallan más bien conectados los unos con los otros a modo de desafíos y reacciones, de procesos y consecuencias no intencionales. Se remiten igualmente a relaciones constituidas en el tiempo y al potencial de diversificación inherente a tales relaciones; a procesos de desarrollo abarcativos y a la complejidad generada por dichos procesos. Este tipo de conclusión deja claro que las siguientes consideraciones no buscan eclipsar la teoría neoinstitucionalista. Por lo contrario, tienen la intención de promover la ampliación y diferenciación de sus conceptos y modelos para hacer más inteligible la interacción intrincada entre el empuje difusor de fuerzas globales omnipresentes y ideologías mundiales por un lado, y, por otro lado, los procesos de adopción, recepción y apropiación dependientes de distintas trayectorias históricas tanto como los mecanismos de recontextualización generadores de desviación y ampliación por parte de diferentes grupos, sociedades, contextos socio-culturales, o civilizaciones.

\section{Mundos de significado culturalmente específicos}

Hemos tendido así el telón de fondo sobre el cual proyectaremos los contornos semánticos del segundo concepto mencionado en el título de este ensayo. Es preciso definirlo igualmente a partir del concepto de "cultura", aunque introduciendo, al menos, otras dos especificaciones. En primer lugar, este concepto - a diferencia de "cultura mundial" - no proviene de un marco teórico que haya sido desarrollado de forma consistente, ni de un marco teórico centrado en los procesos globales de racionalización. "Mundos de significado culturalmente específicos" es una expresión que hace referencia a un concepto que proviene genuinamente de la investigación comparada. Su significado

15 (SCHRIEWER, 2007, p. 402ss). Véanse también (SCHRIEWER, 2005). 
básico puede extraerse, por lo pronto, de la evolución semántica del término "cultura". Tal como lo muestran los análisis de la sociología del conocimiento, el concepto "cultura" experimentó, desde finales del siglo XVIII, un cambio en su significado. De un principio de clasificación del mundo de los objetos pasó a ser un principio de observación, descripción y atribución, que encierra un mayor grado de abstracción y está determinado por dimensiones como la conciencia creciente de la contingencia de las pautas de la vida social, una perspectiva tendiente a relativizar las normas y instituciones sociales y, consecuentemente, una disposición casi natural hacia la comparación (LUHMANN, 1995). Su carácter comparativo se ve continuamente afianzado por descripciones de las vías de desarrollo divergentes - aún en los tiempos de la "thick globalisation" característica del cambio del siglo XX al siglo XXI - de diferentes contextos socioculturales y de las tradiciones de sentido que siguen ejerciendo una influencia culturalmente específica sobre ellos ${ }^{16}$.

En segundo lugar, el concepto de "mundos de significado culturalmente específicos" no se remite solamente, como sería de esperar en vista de las prácticas de observación usuales en la sociología y la educación comparadas, a aspectos estatales o territoriales de las sociedades regionales. Dicho de otro modo, no se refiere, en lo principal, a los condicionamientos político-jurídicos o socioeconómicos. Sin cuestionar la relevancia de aspectos tales, el concepto se refiere preferentemente a dimensiones como las experiencias históricas de diferentes grupos sociales o sociedades enteras, así como también a la manera en la que estas experiencias se ven reflejadas en estructuras simbólicas culturalmente específicas ${ }^{17}$; en las distintas concepciones del tiempo, el cuerpo y la naturaleza de las relaciones sociales ${ }^{18}$; en las imágenes, históricamente consolidadas, de lo propio y lo extraño $;{ }^{19} \mathrm{o}$ en representaciones, modelos de interpretación o "social meanings" arraigados en el conjunto de la sociedad. Nuestro concepto se refiere, pues, a las dimensiones simbólico-ideológico-reflexivas que sirven para dar una asimilación a las experiencias colectivas respecto del orden social y sus transformaciones y rupturas, en otras palabras a experiencias que, a su vez, determinan el accionar social y las decisiones de trascendencia para desarrollos institucio-

${ }^{16}$ Algunos aspectos característicos de la "thick globalisation" se mencionan en (HELD; MCGREW; GOLDBLATT; PERRATON, 1999, p. 21-28 et passim). Respecto del empleo implícito y explícito del concepto de "mundos de significado culturales", véase además, (BADIE, 1992b), así como también (SCHLICHTE, 2005)

17 Cf. (EISENSTADT, 1995), en especial capítulo 7: "Symbolic Structures and Social Dynamics with Special Reference to Studies of Modernization".

${ }^{18}$ Cf., por ejemplo, (COULMAS, 2003) y (TAIRAKO, 2003).

19 Sigue siendo un clásico (ELIAS, 1978), cf. también (SCHMIDT, 1997). 
nales posteriores ${ }^{20}$. Los "mundos de significado culturalmente específicos" se refieren, además, a la semántica histórica (los corpus de saberes acumulados y considerados dignos de ser preservados en las bibliotecas y archivos) generada por las distintas sociedades, al grado de permeabilidad o impermeabilidad - inevitablemente mediado por los idiomas - que estas semánticas poseen, así como al potencial con que cuentan para la producción, constantemente actualizada desde dentro - es decir, autoevolutiva -, de marcos teóricos para la reflexión, saberes interpretativos y significados sociales ${ }^{21}$.

Por último, nuestro concepto se vincula también con investigaciones históricas y reflexiones teóricas que tienen por tema la memoria "colectiva" de etnias, estados y naciones enteras ${ }^{22}$. $\mathrm{Y}$ está relacionado, en parte no menor, con análisis relativos a la actualidad que, en contra de sus propias premisas, no pueden menos que clasificar e interpretar sus resultados - relaciones asombrosamente unívocas entre experiencias políticas, ámbitos lingüísticos y concepciones del mundo fundadas en la religión (o la ideología), por un lado, y variaciones regionales en los valores de orientación, por el otro - bajo la forma de una "geografía específicamente cultural del mundo" (INGLEHART, 1997). En la medida en que reúne e incluye en sí estas y otras dimensiones y facetas interpretativas que, sin importar sus diferentes concretizaciones temáticas, son indicio del carácter propio de las culturas regionales, y de los esquemas socioculturales de producción y asimilación de sentido, el concepto de "mundos de significado culturalmente específicos" se convierte en el verdadero antipolo de la "cultura mundial" neoinstitucionalista.

\section{Más allá de las metáforas: debates teóricos pendientes de solución}

Ambos conceptos - "cultura mundial" y "mundos de significado culturalmente específicos" -, complementarios si bien contrarios, generan en torno suyo un tenso campo de propuestas de investigación y problemas teóricos pendientes, ya que "ni las interrelaciones entre los condicionamientos globales y los desarrollos nacionales y regionales ni el alcance relativo de los primeros y los últimos" (SCHWINN, 2006; cita: p. 16) han sido suficientemente analizados

${ }^{20}$ Cf., por ejemplo, (RINGER, 1979), así como (SCHRIEWER; HARNEY, 1999).

${ }^{21}$ Cf., por ejemplo, los análisis reunidos en (SCHRIEWER; KEINER; CHARLE, 1993), y también el estudio de (KEINER; SCHRIEWER, 2000).

22 Fundamental es (ASSMANN, 1992); continuado en (ASSMANN, 1993). 
en lo que respecta a la investigación comparada empírica. También porque, en relación a lo teórico, el debate que se está dando en el terreno de la sociología, la historia y los estudios regionales entre las "grandes teorías" - las teorías antes mencionadas de la "sociedad mundial", que entienden las diferencias regionales, en esencia, como meros residuos de la historia y, por tanto, déficits y las teorías de la "modernidad múltiple", que abordan tales diferencias como alternativas configurativamente condicionadas - no ha pasado aún de la etapa de un intercambio de argumentos básicos por ambos bandos.

Así, para ilustrar esta tesis por medio de dos ejemplos, la "sociedad mundial" se define, en el contexto de la teoría de la sociedad elaborada por Niklas Luhmann, como correlato directo de una "diferenciación funcional". El esquema de diferenciación de la modernidad, orientado a problematizaciones de relevancia para el conjunto de la sociedad, lleva implícito - según Luhmann - el hecho de que sus sistemas parciales, diferenciados de acuerdo con su función, tienden a extender el alcance de sus operaciones a nivel, en principio, mundial. Cada sistema parcial, por así decirlo, intenta desatar la dinámica que es propia de su función y expandir sus comunicaciones, traspasando ámbitos y barreras. Esta dinámica puede observarse, por ejemplo, bajo la forma del comercio internacional y la interconexión de las bolsas de valores a nivel mundial, en los congresos y publicaciones científicas internacionales, en una cooperación tecnológica de alcance mundial o, como decíamos, en la difusión transcontinental de innovaciones en materia de organización educativa. Desde una perspectiva evolutiva, entre una forma de diferenciación social orientada primordialmente por funciones y la sociedad mundial emergente existe - de acuerdo con Luhmann - una relación de apoyo y fortalecimiento mutuos. Sin embargo, tampoco pasa inadvertido para Luhmann el hecho de que hay notables diferencias en el modo en que distintas sociedades participan de los lineamientos estructurales típicos de la sociedad mundial. En virtud de constelaciones que varían de región a región, la formación de estructuras de diferenciación funcional puede resultar favorecida en un caso dado, mientras que puede verse dificultada en otro, y en otro más limitarse de manera selectiva o adoptarse de modo sólo parcial, mediante estrategias arbitrarias de aceptación y subversión. No obstante la preponderancia primordial que atribuye a las estructuras de diferenciación funcional e interconexión mundial de la sociedad, esta teoría ofrece, pues, un conjunto de herramientas conceptuales que hacen posible también el análisis de la dinámica de los procesos de diversificación que siguen verificándose (e incluso potenciándose) en tiempos de un entrelazamiento creciente a nivel global. Es aquí donde conviene ubicar conceptos tales como "autodeterminación para el cambio", "acentuación de la divergencia" y "condicionamiento", que hacen referencia a mecanismos de creación o potenciación de diferencias que, partiendo de "condicionamientos 
particulares" contingentes - de diferencias de tipo local, regional, geográfico o cultural -, ponen en movimiento cadenas de efectos no pronosticables. Estos "condicionamientos" aparecen siempre que el modo específico de operación de un sistema parcial funcional se topa con condiciones iniciales diferentes en una región dada, se conecta con éstas de manera selectiva y las transforma y utiliza para la construcción y consolidación de sistemas funcionales. Son también estas interacciones variables entre los imperativos funcionales de los sistemas parciales de tipo social y los condicionamientos particulares dominantes en una región dada las que terminan por producir los modelos divergentes de estructuración que resultan en "transformaciones extremadamente desiguales dentro del marco de la sociedad mundial". Así pues, los mecanismos de creación y potenciación de diferencias generan múltiples transformaciones que - según una lacónica expresión de Luhmann - conceden a las regiones "las oportunidades de un destino propio" (LUHMANN, 2006, p. 643).

Desde el bando opuesto, Shmuel N. Eisenstadt ha elaborado un modelo del complejo entramado de componentes y fases procesuales, dejando estrictamente de lado las premisas de los conceptos de la "convergencia". Según su tesis, este modelo resultó determinante en la formación de los distintos tipos de modernidad (EISENSTADT, 2000; SACHSENMAIER; RIEDEL; EISENSTADT, 2002). Haciendo uso de numerosos estudios de historia cultural comparada, Eisenstadt asigna a los sistemas religiosos, es decir, a los ordenamientos simbólicos e interpretaciones de la historia y el mundo de concepción ultramundana, un papel decididamente preponderante en la conformación de lo que él denomina "programas culturales" específicos de una sociedad o cultura dada. Pero estas dimensiones de la vida social no operan en forma aislada ni inmediata. Se encuentran, antes bien, instaladas en una compleja red de interrelación en la que operan también, de manera distinta en cada caso, dimensiones culturales (aparte de componentes religiosos y simbólicos, también, por ejemplo, concepciones jurídicas y experiencias históricas), dimensiones estructurales (diferenciación social, industrialización, urbanización) y aspectos institucionales (las formas en las que se plasman las concepciones modernas del estado y la actividad económica capitalista). Es partiendo de tales premisas que puede verse que los encuentros entre los programas de la modernidad europea y las sociedades extraeuropeas no fueron - ni son - unidireccionales. Son origen, más bien, de un sinnúmero de selecciones, interpretaciones y apropiaciones, cuyos marcos de referencias están instalados en la historia y en las experiencias históricas de las colectividades socioculturales. Es a partir de tales procesos interpretativos de interacción y transformación que, según Eisenstadt, han surgido versiones modificadas de los programas culturales y políticos de la modernidad, que 
encierran en ocasiones un reacomodamiento del peso de sus componentes o incluso antinomias entre ellos. Lo mismo puede aplicarse a la reconstrucción de identidades colectivas e imágenes de lo propio, o a la diferenciación de reordenamientos institucionales. Esto no significa, sin embargo, que las sociedades modernas no hayan desarrollado también trayectorias convergentes y problemáticas análogas en campos de acción de extrema relevancia para la sociedad. Tal evolución puede observarse claramente en la estructura de la industria y el empleo, en la planificación urbana y en los sistemas educativos. No obstante, la forma en que se han abordado dichas problemáticas, incluyendo los recursos semánticos y esquemas de procesamiento de sentido utilizados en la discusión y en la reflexión en torno a reformas, resulta divergente en función de la dinámica de la modernidad que se haya conformado históricamente en distintas sociedades o culturas.

Más allá de lo brillante de estas propuestas o, precisamente, dada su amplísima pretensión de validez, lo que no ha logrado establecerse (todavía) son intentos teóricos de intermediación o integración. Mejor dicho, se requiere de modelos que estén construidos de manera más compleja sin dejar de lado criterios de consistencia conceptual; que resulten apropiados para explicar los resultados de la sociología y la educación comparada internacional que hemos resumido anteriormente: la presencia, simultánea y llena de contrastes, de tendencias de interconexión a nivel mundial y estructuras generadoras de divergencia, con sus entrecruzamientos, superposiciones e hibridaciones.

De allí resalta el papel desempeñado por las investigaciones comparadas, el cual no consiste en profundizar la discusión acerca de una teoría sociológica general. Son relevantes, más bien, los promisorios intentos de una historización de los procesos de mundialización que están llevándose a cabo en el marco de la Global History, una disciplina que está tomando cada vez más forma. A la luz de cuestionamientos y perspectivas de investigación de carácter novedoso, estos estudios indagan los procesos de encuentro, migración y/o superposición de culturas, incluso los más alejados históricamente ${ }^{23}$. Así logran inyectar "una tradición narrativa al estudio de la globalización", tal como lo ha recomendado recientemente Dominic Sachsenmaier a modo de programa paliativo para la diferenciación de las grandes propuestas teóricas (SACHSENMAIER, 2006, p. 465). Y ello porque la historización representa, irrecusablemente, la empirización de "expresiones de moda y diagnósticos de altos vuelos"24. Para investigaciones comparadas que se dedican a esa empirización no se carece de bases sistemáti-

${ }^{23}$ Véase, por ejemplo, (HOPKINS, 2002; OSTERHAMMEL; PETERSSON, 2003; BAYLY, 2004; GRUZINSKI, 2004).

${ }^{24}$ Tal la formulación de (SCHWINN, 2005, p. 208). 
cas. Es más: parece estar formándose, entre las distintas perspectivas teóricas, un consenso que establece que todo nuevo progreso en nuestros conocimientos debe presuponer un plano medio de teoremas y conceptos acerca de la globalización (SCHWINN, 2005, p. 208ss.; OSTERHAMMEL; PETERSSON, 2003; SACHSENMAIER, 2006). Nos referimos a teoremas y conceptos tales como - sin pretender ser exhaustivos -: los movimientos migratorios transnacionales y las actividades de las diásporas dislocadas; los mecanismos, agentes, formas y canales de los procesos de difusión y recepción; la expansiva fuerza estructuradora del principio de orden de la diferenciación funcional; la intensificación recíproca de interacciones (su alcance, duración y frecuencia) y redes (según su densidad, centralidad y medios); el desarrollo de técnicas de comunicación e información autonomizadas; la creciente cantidad e importancia de las organizaciones internacionales; la generalización de observaciones y referencias entre estados; la construcción (que esta última implica) de horizontes de comparación y escalas de modernidad que acentúan la competencia entre naciones; finalmente, la fuerza interpretativa que sigue residiendo en las semánticas sedimentadas en las lenguas nacionales.

\section{REFERENCIAS}

ANDERSON-LEVITT, Kathryn M. (Comp.). Local Meanings and Global Schooling. Anthropology and World Culture Theory. Nueva York: Palmgrave Macmillan, 2003.

ASSMANN, Aleida. Arbeit am nationalen Gedächtnis. Eine kurze Geschichte der deutschen Bildungsidee. Fráncfort del Meno \& París: Campus \& Editions de la Maison des Sciences de l'Homme, 1993.

ASSMANN, Jan. Das kulturelle Gedächtnis: Schrift, Erinnerung und politische Identität in frühen Hochkulturen. Múnich: Beck, 1992.

BADIE, Bertrand. Analyse comparative et sociologie historique. Revue Internationale des Sciences Sociales, n. 133, 1992a.

1992b.

. L'état importé. Essai sur l'occidentalisation de l'ordre politique. París: Fayard,

BAYLY, Christopher A. The Birth of the Modern World 1780-1914. Global Connections and Comparisons. Oxford: Blackwell, 2004.

BERGER, Peter L.; LUCKMANN, Thomas. La Construcción social de la realidad. Buenos Aires: Amorrortu, 1976. 
BOLI, John. New Citizens for a New Society. The Institutional Origins of Mass Schooling in Sweden. Oxford: Pergamon, 1989.

BOLI, John; RAMIREZ, Francisco O. World Culture and the Institutional Development of Mass Education. En: RICHARDSON, John G. (Comp.). Handbook of Theory and Research for the Sociology of Education. Nueva York: Greenwood, 1986. p. 65-90.

BOLI, John; THOMAS, George M. (Comps.). Constructing World Culture. International Nongovernmental Organizations since 1875. Stanford, CA: Stanford University Press, 1999.

BRUNO-JOFRÉ, Rosa; SCHRIEWER, Jürgen (Comps.). The Global Reception of John Dewey's Thought. Multiple Refractions Through Time and Space. New York \& London: Routledge, 2012.

CARUSO, Marcelo. Geist oder Mechanik. Unterrichtsordnungen als kulturelle Konstruktionen in Preußen, Dänemark (Schleswig-Holstein) und Spanien 1800-1870. Komparatistische Bibliothek. Francfort del Meno : Peter Lang, 2010. v. 19.

CLARK, Ian. Globalization and Fragmentation. International relations in the twentieth century. Oxford: Oxford University Press, 1997.

COULMAS, Florian. Die Kultur Japans: Tradition und Moderne. Múnich: Beck, 2003.

DEWEY, John. Procesos de recepción y adopción en diferentes espacios socio-históricos. En: BRUNO-JOFRÉ, Rosa; SCHRIEWER, Jürgen. Encounters on education/Encuentros sobre educación/ Rencontres sur l'éducation. Kingston, Ontario: Queen's University, Faculty of Education, Kingston, Ontario: Queen's University, Faculty of Education, número especial, v. 10, fall/otoño/automne 2009.

DRORI, Gili S.; MEYER, John W.; RAMIREZ, Francisco O.; SCHOFER, Evan. Science in the Modern World Polity. Institutionalization and Globalization. Stanford, CA: Stanford University Press, 2003.

EISENSTADT, Shmuel N. Die Vielfalt der Moderne. Weilerswist: Velbrück, 2000.

. Power, Trust, and Meaning. Essays in Sociological Theory and Analysis. Chicago/ Londres: Chicago University Press, 1995.

ELIAS, Norbert. Über den Prozess der Zivilisation, T. 1. Fráncfort del Meno: Suhrkamp, 6. Aufl., 1978.

FIALA, Robert; LANFORD, Audri Gordon. "Educational Ideology and the World Educational Revolution, 1950-1970”. En: Comparative Education Review, n. 31, p. 315-332, 1987.

FRIEDMAN, Jonathan. Cultural Identity and Global Process. Londres: Sage Publications, 1994. 
FUCHS, Eckhardt; SCHRIEWER, Jürgen (Comps.). "Internationale Organisationen als Global Players in Bildungspolitik und Pädagogik". Número extraordinario de: Zeitschrift für Pädagogik, v. 53, n. 2, p. 145-223, 2007.

GREEN, Andy; WOLF, Alison; LENEY, Tony. Convergence and Divergence in European Education and Training Systems. Bedford Way Papers. Londres: University of London, Institute of Education, 1999.

GRUZINSKI, Serge. Les quatre parties du monde. Histoire d'une mondialisation. París: Editions de la Martinière, 2004.

HASSE, Raimund; KRÜCKEN, Georg. Neo-Institutionalismus. $2^{\text {a }}$ impresión aumentada. Bielefeld: transcript Verlag, 2005.

HELD, David; MCGREW, Anthony; GOLDBLATT, David; PERRATON, Jonathan. Global Transformations. Politics, Economics and Culture. Cambridge: Polity Press, 1999.

HOPKINS, Antony G. (Comp.). Globalization in World History. Nueva York \& Londres: Norton \& Co., 2002.

INGLEHART, Ronald. Modernization and Postmodernization: Cultural, Economic, and Political Change in 43 Societies. Princeton, NJ: Princeton University Press, 1997.

JÜRGENS, Ulrich. Convergence or Divergence? Production Systems and Industrial Relations in Europe. The 21st Century Forum: The European Integration and Japan. The Bulletin of Yokohama City University, v. 52, n. 2, p. 1-39, 2001.

KEINER, Edwin; SCHRIEWER, Jürgen. Erneuerung aus dem Geist der eigenen Tradition? Über Kontinuität und Wandel nationaler Denkstile in der Erziehungswissenschaft. Revue Suisse des Sciences de l'éducation, v. 22, n. 1, p. 27-50, 2000.

KRÜCKEN, Georg; DRORI, Gili S. (Comps.). World Society. The Writings of John Meyer. Oxford etc.: Oxford University Press, 2009.

LANGLOIS, Simon. Convergence or Divergence? Comparing Recent Social Trends in Industrial Societies. Fráncfort del Meno, Montréal, Kingston etc.: Campus \& McGill-Queen's University Press, 1994.

LUHMANN, Niklas. Kultur als historischer Begriff. En: Gesellschaftsstruktur und Semantik. Studien zur Wissenssoziologie der modernen Gesellschaft, Tomo 4. Fráncfort del Meno: Suhrkamp, 1995. p. 31-54.

. La sociedad de la sociedad. México: Herder y Universidad Iberoamericana, 2006. p. 108ss. y 639ss.

MAYER, Karl Ulrich. The paradox of global social change and national path dependencies. Life course pattern in advanced societies. En: WOODWARD, Alison; Kohli, Martin (Comp.). Inclusions and Exclusions in European Societies. Londres/Nueva York: Routledge, 2001. p. 89-110. 
MENZEL, Ulrich. Globalisierung versus Fragmentierung. Fráncfort del Meno: Suhrkamp, 1998.

MEYER, John W.; KAMENS, David H.; BENAVOT, Aaron. School Knowledge for the Masses: World Models and National Primary Curricular Categories in the Twentieth Century. Washington/Londres: Falmer Press, 1992.

MEYER, John W.; RAMIREZ, Francisco O. La educación en la sociedad mundial. Teoría institucional y agenda de investigación de los sistemas educativos contemporáneos. Barcelona: Octaedro, 2011.

. La institucionalización mundial de la educación. En: SCHRIEWER, Jürgen (Comp.). Formación del discurso en la educación comparada. Barcelona: Ediciones Pomares, 2002. p. 91-111.

OSTERHAMMEL, Jürgen; PETERSSON, Niels P. Geschichte der Globalisierung. Dimensionen, Prozesse, Epochen. Múnich: Beck, 2003.

PALOMBA, Donatella (Comp.). Changing Universities in Europe and the "Bologna Process". Rome: Aracne, 2008.

RAMIREZ, Francisco O.; BOLI-BENNETT, John. The Political Construction of Mass Schooling: European Origins and Worldwide Institutionalization. En: Sociology of Education, n. 60, p. 2-17, 1987.

RINGER, Fritz K. Education and Society in Modern Europe. Bloomington: Indiana University Press, 1979.

ROTHERMUND, Dietmar (Comp.). Aneignung und Selbstbehauptung: Antworten auf die europäische Expansion. Múnich: Oldenbourg, 1999.

SACHSENMAIER, Dominic. Global history and critiques of western perspectives. Comparative Methodologies in the Social Sciences - Cross-Disciplinary Inspirations, comp. por Jürgen Schriewer. Núm. especial de Comparative Education, v. 42, n. 3, p. 451-470, 2006.

SACHSENMAIER, Dominic; RIEDEL, Jens; EISENSTADT, Shmuel N. (Comps.). Reflections on Multiple Modernities. European, Chinese and Other Interpretations. Leiden etc.: Brill, 2002.

SCHLICHTE, Klaus. Der Staat in der Weltgesellschaft. Politische Herrschaft in Asien, Afrika und Lateinamerika. Fráncfort del Meno: Campus, 2005.

SCHMIDT, Alexander. Reisen in die Moderne. Der Amerika-Diskurs des deutschen Bürgertums vor dem Ersten Weltkrieg im europäischen Vergleich. Berlín: Akademie Verlag, 1997.

SCHRIEWER, Jürgen. Internacionalidades múltiples: surgimiento de una ideología à nivel mundial y persistencia de visiones idiosincráticas mundiales. En: CHARLE, Christophe; SCHRIEWER, Jürgen; WAGNER, Peter (Comps.). Redes Intelectuales 
trasnacionales: Formas de conocimiento académico y búsqueda de identidades culturales. Barcelona y México: Pomares, 2007. p. 359-408.

. El método comparativo y la necesidad de externalización: Criterios metodológicos y conceptos sociológicos. En: SCHRIEWER, Jürgen; PEDRÓ, Francesc. Educación Comparada: Teorías, Investigaciones, Perspectivas, Edición. Barcelona: Promociones y Publicaciones Universitarias, 1993. p. 189-251.

. "Rationalized Myths" in European Higher Education. The Construction and Diffusion of the Bologna Model. European Education, v. 41, n. 2, p. 31-51, 2009.

. Sistema mundial y redes de interrelación: la internacionalización de la educación y el papel de la investigación comparada. In: CARUSO, Marcelo; TENORTH, Heinz-Elmar (Comps.). Internacionalización. Políticas educativas y reflexión pedagógica en un medio global. Buenos Aires: Granica, 2011. p. 41-105.

. Wie global ist institutionalisierte Weltbildungsprogrammatik? Neo-institutionalistische Thesen im Licht kulturvergleichender Analysen. En: HEINTZ, Bettina; MÜNCH, Richard; TYRELL, Hartmann (Comps.). Weltgesellschaft. Theoretische Zugänge und empirische Problemlagen. Stuttgart: Lucius \& Lucius, 2005. p. 415-441.

SCHRIEWER, Jürgen; CARUSO, Marcelo (Comps.). Nationalerziehung und Universalmethode - frühe Formen schulorganisatorischer Globalisierung. Leipzig: Leipziger Universitätsverlag, 2005. p. 7ss.

SCHRIEWER, Jürgen; HARNEY, Klaus Beruflichkeit versus Culture technique: Pour une sociogenèse de la sémantique du travail en France et en Allemagne. En: DIDRY, Claude; WAGNER, Peter; ZIMMERMANN, Bénédicte. Le Travail et la Nation. Histoire croisée de la France et de l'Allemagne à l'horizon européen. Paris: Editions de la Maison des Sciences de l'Homme, 1999. p. 107-146.

SCHRIEWER, Jürgen; KEINER, Edwin; CHARLE, Christophe (Comps.). Sozialer Raum und akademische Kulturen / A la recherche de l'espace universitaire européen. Fráncfort del Meno: Peter Lang, 1993.

SCHRIEWER, Jürgen; MARTINEZ, Carlos. Ideología educativa mundial o reflexión idiosincrática? El discurso pedagógico en España, Rusia (Unión Soviética) y China del siglo XX. Revista de Educación, Madrid, n. 343, p. 531-577, mayo-ago. 2007.

SCHWINN, Thomas. Die Vielfalt und die Einheit der Moderne - Perspektiven und Probleme eines Forschungsprogramms. En: Die Vielfalt und die Einheit der Moderne. Kultur - und strukturvergleichende Analysen. Wiesbaden: Verlag für Sozialwissenschaften, 2006.

. Weltgesellschaft, multiple Moderne und die Herausforderungen für die soziologische Theorie. En: HEINTZ, Bettina; MÜNCH, Richard; TYRELL, Hartmann (Comps.). Weltgesellschaft. Theoretische Zugänge und empirische Problemlagen. Stattgart: Lucius \& Lucius, 2005 (=Zeitschrift für Soziologie, Sonderheft 1). p. 205-222.

SPYBEY, Tony. Globalization and World Society. Cambridge: Polity Press, 1996. 
STEINER-KHAMSI, Gita. Essays Review. Globalisation, Societies and Education, v. 3, n. 2, p. 229-237, 2005.

STRANG, David; MEYER, John W. "Institutional Conditions for Diffusion”. Theory and Society, n. 22, p. 487-511, 1993.

STROMQUIST, Nelly P.; MONKMAN, Karen (Comps.). Globalization and Education. Integration and Contestation across Cultures. Lanham, Maryland: Rowman \& Littlefield, 2000 .

TAIRAKO, Tomonaga. Zeitlichkeit und Räumlichkeit im Hinblick auf die traditionelle japanische Zeitmessung. Hitotsubashi Journal of Social Studies, v. 35, n. 2, p. 47-62, 2003.

THOMAS, George M.; MEYER, John W.; RAMIREZ, Francisco O.; BOLI, John. Institutional Structure. Constituting State, Society, and the Individual. Newbury Park, CA: Sage, 1987.

WALLERSTEIN, Immanuel. The modern world-system. Nueva York: Academic Press, 1974-1989. v. I-III. 2004.

.World-systems analysis: an introduction. Durham, N.C.: Duke University Press,

Texto recebido em 23 de julho de 2013.

Texto aprovado em 23 de julho de 2013. 